\title{
Preoperative Factors Associated With Antiepileptic Drug Withdrawal Following Surgery for Intractable Temporal Lobe Epilepsy
}

\author{
Taketoshi MAEHARA ${ }^{1}$ and Kikuo OHNO ${ }^{1}$ \\ ${ }^{1}$ Department of Neurosurgery, Tokyo Medical and Dental University, Tokyo
}

\begin{abstract}
The outcomes of postoperative antiepileptic drug (AED) cessation after temporal lobe epilepsy surgery were investigated to identify the preoperative factors associated with seizure-free status without drug treatment in 40 patients who underwent resective epilepsy surgery between 2000 and 2007 for the treatment of complex partial seizures of the type typically associated with temporal lobe epilepsy. All patients were followed up for at least 3.5 years after surgery. After the numbers and types of AEDs used as preoperative and postoperative treatments were determined, the patients who obtained seizure-free outcome for at least 2 years after AED cessation were analyzed. Sex, age at surgery, age at seizure onset, time to operation, seizure frequency, operation side, invasive study, pathological types (hippocampal sclerosis or others), history of generalized convulsive seizures, and number of preoperative AEDs were analyzed as factors potentially associated with seizure-free status without drug treatment. In total, $\mathbf{3 5 \%}$ of the patients who underwent temporal lobe epilepsy surgery achieved seizure-free status without drugs. The mean number AEDs fell from 2.1 preoperatively to 1.2 after the surgery. Seizures recurred in $18 \%$ of the patients after AED withdrawal. A shorter duration from seizure onset to surgery was found to be significantly related to successful withdrawal of postoperative AED treatment. Our results suggested that early surgical intervention after seizure onset is an important precondition for achieving seizure-free status without drugs after temporal lobe epilepsy surgery.
\end{abstract}

Key words: temporal lobe epilepsy, discontinuation, antiepileptic drug, epilepsy surgery, seizure freedom

\section{Introduction}

Patients with temporal lobe epilepsy (TLE) refractory to medical treatment are the most common candidates for surgical intervention. According to a recent review, seizure-free status is achieved in $70 \%$ of patients after temporal lobectomy. ${ }^{8}$ This high rate of success suggests that important benefits can be gained from the introduction of resective surgery methods for patients suffering from TLE. Patients with TLE who undergo surgery hope to become seizure-free, but also to be completely cured and to discontinue antiepileptic drugs (AEDs). ${ }^{13)}$ While several recent papers have discussed this issue, ${ }^{1,4-7,12)}$ there have been difficulties in assessing the rates or predictors of postoperative drug treatment. Differences in the postoperative AED reduction criteria, pathologies, and medico-social en-

Received December 16, 2010; Accepted January 28, 2011 vironments among the published reports create considerable difficulties for this type of assessment. ${ }^{1,4,6,9)}$ In spite of the analytical challenges these differences inevitably pose, postoperative AED reduction affects the patient's quality of life and levels of disability. ${ }^{2,3,12)}$ Patients considering surgical intervention usually ask whether life will be possible without seizures or AEDs after the surgery. In the course of presurgical counseling, physicians need to provide these patients with adequate information on postoperative AED reduction and the predictors for successful AED cessation.

In this study, we reviewed the rate of postoperative AED cessation in patients after TLE surgery, and tried to identify the preoperative factors associated with seizure-free status without drug treatment.

\section{Materials and Methods}

This study included 40 patients who underwent resective epilepsy surgery between 2000 and 2007 
for the treatment of complex partial seizures of the type typically associated with TLE. All patients were referred to our epilepsy outpatient clinic for surgical treatment. The patients underwent resective surgery for TLE after presurgical magnetic resonance (MR) imaging, fluorodeoxyglucose positron emission tomography, repeated electroencephalography (EEG), video-EEG monitoring, and neuropsychological testing.

The patients were followed up for at least 3.5 years after surgery. The numbers and types of AEDs used as preoperative and postoperative treatments were first analyzed. Then patients who obtained seizure-free outcome for at least 2 years after AED withdrawal were identified. Factors potentially associated with seizure-free status without drug treatment were considered to be sex, age at surgery, age at seizure onset, time to operation, seizure frequency, operation side, invasive study, pathological type (hippocampal sclerosis [HS] or others), history of generalized convulsive seizures, and number of preoperative AEDs. Seizure frequency was divided into high ( $>4$ seizures/month) and low groups.

Seventeen of 40 patients were monitored by chronic electrocorticography (ECoG) and all 40 patients were monitored by intraoperative ECoG. Intraoperative hippocampal or parahippocampal ECoGs were recorded to judge whether hippocampectomy was indicated. Twenty patients with HS and 10 patients without HS underwent anteromedian temporal lobectomy. ${ }^{10,11)}$ Ten patients with normal hippocampus underwent lesionectomy and focus resection by sparing hippocampectomy (Table 1).

The protocol for AED reduction was to consider drug withdrawal when seizure-free status was maintained for at least 1 year after surgery, provided that the patient desired AED reduction and agreed to accept the risk of seizure recurrence. AEDs were also discontinued at 1 year after surgery in 1 female patient who strongly desired to discontinue the AEDs for pregnancy. The AEDs were gradually tapered, one by one. If a patient experienced auras or seizures, AED administration was continued at the minimum doses necessary for stopping seizures without further tapering. Before discontinuing the final AED altogether, absence of epileptic discharges on EEG was reconfirmed and the patient was asked to reconfirm his or her desire to discontinue the drug in spite of the risk of seizure recurrence and potential effects on quality of life.

Correlations between presurgical factors and postoperative AED cessation were evaluated by either the t-test for continuous variables or the chisquare test for categorical variables. Correlations
Table 1 Characteristics of patients

\begin{tabular}{ll}
\hline \multicolumn{1}{c}{ Characteristics } & \multicolumn{1}{c}{$\begin{array}{c}\text { Number or } \\
\text { means } \pm \text { SD }\end{array}$} \\
\hline Sex (male/female) & $16 / 24$ \\
Age at surgery, yrs & $9-59(30.0 \pm 11.9)$ \\
Age at seizure onset, yrs & $3-57(15.3 \pm 9.3)$ \\
Time to surgery, yrs & $1-40(14.7 \pm 11.9)$ \\
Side (left/right) & $21 / 19$ \\
Seizure frequency (high*/low) & $21 / 19$ \\
Invasive study & 17 \\
Pathological type & \\
$\quad$ HS & 20 \\
Others & 20 \\
$\quad$ tumor & 12 \\
$\quad$ vascular** & \multicolumn{2}{c}{6} \\
$\quad$ normal & 2 \\
History of generalized convulsive seizures & 14 \\
Preoperative AEDs & \\
monotherapy & 12 \\
polytherapy & 28 \\
Surgical outcome (Engel Class) & \\
I & 33 \\
II & 3 \\
III & 4 \\
IV & 0 \\
Postoperative follow up, yrs & $3.6-10.4(6.8 \pm 2.1)$ \\
Follow up after AED discontinuation, yrs & $2.0-6.2(4.6 \pm 1.3)$ \\
\hline
\end{tabular}

*Seizure frequency high: $>4$ seizures/month. ${ }^{* *}$ Vascular: vascular lesions including cavernous angiomas and arteriovenous malformations. AED: antiepileptic drug, HS: hippocampal sclerosis, SD: standard deviation.

were considered significant at $\mathrm{p}<0.05$.

\section{Results}

Table 1 summarizes the clinical characteristics of the patients. Among the 40 patients, 33 obtained Engel Class I outcome and 14 discontinued AEDs. The patients were followed up for 3.6-10.4 years (mean 6.8 years) after surgery. AEDs were completely discontinued in 17 patients, and 3 of these 17 experienced recurrent seizures at 2, 4, and 19 months. These 3 patients were treated by AED therapy and became seizure-free again. Thus, the successful AED discontinuation rate was $82 \%(14 / 17)$ in the present series. The time to discontinuation from the reduction of AEDs was 0.3-4.4 years (mean 2.2 years). After AED cessation, the patients were followed up for 2.0-6.2 years (mean 4.6 years).

The mean number of AEDs was reduced from 2.1 preoperatively to 1.2 postoperatively. Before the operation, 13 patients received mono AED and 28 patients received multiple AEDs $(2$ AEDs in 13 patients, 3 AEDs in 13 patients, and 4 AEDs in 2 patients). Carbamazepine was the most frequently administered AED before surgery, and zonisamide 
Table 2 Pre- and postoperative antiepileptic drugs

\begin{tabular}{|c|c|c|c|c|c|c|c|c|c|c|}
\hline \multirow{2}{*}{ Surgical outcome } & \multirow{2}{*}{$\begin{array}{l}\text { Number of } \\
\text { patients }\end{array}$} & \multicolumn{9}{|c|}{ Pre-/postoperative antiepileptic drugs } \\
\hline & & CBZ & ZNS & PHT & VPA & CLB & CZP & $\mathrm{PB}$ & PRM & TPM \\
\hline Engel Class I & 33 & $11 / 0$ & $6 / 0$ & $2 / 0$ & $0 / 0$ & $1 / 0$ & $1 / 0$ & $0 / 0$ & $0 / 0$ & $0 / 0$ \\
\hline off AED & 14 & $15 / 16$ & $10 / 5$ & $7 / 5$ & $5 / 3$ & $2 / 4$ & $4 / 1$ & $2 / 1$ & $0 / 0$ & $0 / 0$ \\
\hline with AED & 19 & $7 / 7$ & $0 / 0$ & $4 / 4$ & $1 / 0$ & $1 / 1$ & $1 / 1$ & $1 / 0$ & $2 / 2$ & $0 / 1$ \\
\hline Engel Classes II and III & 7 & $33 / 23$ & $16 / 5$ & $13 / 9$ & $6 / 3$ & $4 / 5$ & $6 / 1$ & $3 / 1$ & $2 / 2$ & $0 / 1$ \\
\hline
\end{tabular}

CBZ: carbamazepine, CLB: clobazam, CZP: clonazepam, PB: phenobarbital, PHT: phenytoin, PRM: primidone, TPM: topiramate, VPA: valproate, ZNS: zonisamide.

Table 3 Factors related to antiepileptic drug (AED) discontinuation

\begin{tabular}{|c|c|c|c|}
\hline & Without AED & With AED & Probability \\
\hline Sex (male/female) & $6 / 8$ & $10 / 16$ & 0.79 \\
\hline Age at surgery, yrs & $26.8 \pm 13.4$ & $31.1 \pm 11.1$ & 0.37 \\
\hline Age at seizure onset, yrs & $18.6 \pm 13.4$ & $13.0 \pm 5.3$ & 0.06 \\
\hline Time to surgery, yrs & $9.0 \pm 11.7$ & $17.8 \pm 10.7$ & 0.01 \\
\hline Side (left/right) & $8 / 6$ & $14 / 12$ & 0.84 \\
\hline Seizure frequency (high*/low) & $6 / 8$ & $15 / 11$ & 0.37 \\
\hline Invasive study & 7 & 10 & 0.97 \\
\hline Pathological type & & & 0.18 \\
\hline HS & 5 & 15 & \\
\hline Others & 9 & 11 & \\
\hline tumor & 7 & 5 & \\
\hline vascular** & 2 & 4 & \\
\hline normal & 0 & 2 & \\
\hline $\begin{array}{l}\text { History of generalized convulsive } \\
\text { seizures }\end{array}$ & 5 & 9 & 0.94 \\
\hline Preoperative AEDs & & & 0.15 \\
\hline monotherapy & 7 & 7 & \\
\hline polytherapy & 7 & 19 & \\
\hline
\end{tabular}

${ }^{*}$ Seizure frequency high: $>4$ seizures/month. ${ }^{* *}$ Vascular: vascular lesions including cavernous angiomas and arteriovenous malformations. HS: hippocampal sclerosis.

was the second most frequently administered AED. In patients who obtained Engel Class I outcome, the mean number of AEDs given was reduced from 2.1 before surgery to 1.1 after surgery (Table 2).

Statistical analysis revealed that AED cessation was significantly associated with short time to surgery $(p=0.01)$. There were no associations with sex, age at surgery, age at seizure onset, operation side, seizure frequency, invasive study, pathological type, mono- or polytherapy, or history of generalized convulsive seizures (Table 3 ).

Eight of the 18 patients with lesions (12 tumors and 6 vascular lesions) had hippocampal spikes on ECoG and underwent hippocampectomy. The hippocampus was spared in 10 patients with normal hippocampus on MR imaging, 8 of whom had normal hippocampal ECoG and 2 of whom had hip- pocampal spikes on ECoG (both elderly). There were no statistically significant associations between AED cessation and hippocampectomy $(\mathrm{p}=0.81)$ or hippocampal spikes on ECoG ( $p=0.37)$.

\section{Discussion}

The ultimate goals of epilepsy surgery are complete resection of the epileptogenic areas, prevention of seizures, and elimination of any need for AED treatment. In this study, $35 \%$ of the patients who underwent TLE surgery achieved seizure-free status without AED treatment, and the mean number of AEDs was reduced from 2.1 preoperatively to 1.2 after the surgery. Seizures recurred in $18 \%$ of patients after AED cessation. Our study also indicated that a shorter duration from seizure onset to sur- 
gery was significantly associated with successful postoperative AED cessation. Several other recent studies have reported similar findings of seizurefree, drug-free status in $22 \%$ to $42 \%$ of patients after temporal lobe resection. ${ }^{1,4-7,12)}$ Differences in pathologies, surgical procedures, and AED reduction policies in these other studies can explain the differences in the reported rates of postoperative AED cessation.

Several previous studies focused on differences in the AED cessation rate between HS and other pathologies, including vascular malformations, tumors, cysts, cortical dysplasia, and normal hippocampus. ${ }^{5,6,12)}$ Postoperative AED cessation was observed in $22 \%$ of patients confined to HS. ${ }^{6)}$ The rate of AED cessation was lower in patients with HS than in patients with other pathologies. ${ }^{12)}$ Specifically, $27.8 \%$ of patients with HS and $45.9 \%$ of patients with other lesions managed to successfully discontinue AEDs 1 to 5 years after surgery. These results are very similar to our findings, in which $25 \%$ of patients with $\mathrm{HS}$ and $45 \%$ of patients with other pathologies obtained seizure-free status without drug treatment (no significant difference). Another study ruled out the statistical importance of HS as a predictive factor of AED. ${ }^{5)}$ Patients with HS tended to have a low rate of postoperative AED discontinuation, but not to a significant extent.

Our study also investigated the effects of the surgical procedures employed in the temporal lobe resections. The procedures included anterior temporal lobectomy (ATL), selective amygdala hippocampectomy (AHE), and lesionectomy with or without hippocampal resection. Only a few studies have focused on the relationships between surgical procedures and the overall rate of seizure-free outcome without drugs. ${ }^{1,7)}$ The type of surgery (ATL, AHE, or lesionectomy) was not a predictive factor in patients with seizure recurrence after AED cessation. ${ }^{1)}$ Similarly, in our study, hippocampectomy was not a predictive factor if the focus was correctly identified. We also found that a smaller cortical resection in the left dominant side versus the right side had no significant predictive value. Analysis of the surgical outcome and the amount of neocortex resection in anterior temporal lobectomy found that the postoperative seizure-free status was unchanged. ${ }^{7)}$ These results suggest that the surgical procedures employed have little influence on the rate of successful AED cessation.

The policies and methods for tapering and ceasing AEDs have varied from study to study. Few studies have actually reported protocol-based policies for drug reduction. ${ }^{1,5)}$ Our group first required 1-year seizure-free status after surgery, then tapered the
AEDs gradually. Our analyses may have excluded patients who were able but reluctant to stop AEDs, as we only stopped the AEDs if the patients gave consent. The physician's advice and patient's consent are both important for AED cessation, as postoperative seizure-free patients often fear that seizure will recur after their drugs are withdrawn. ${ }^{4}$ Seizure recurrence after AED cessation is important to consider in setting the drug-reduction protocol. The successful AED cessation rate in the present series was $82 \%$, with a mean follow up of 4.6 years, which is very close to the rates reported in other recent studies ${ }^{4,5,7,12)}$ and slightly exceeded that reported in a review of seizure recurrence after planned AED cessation (seizures recurred in an average of $33.8 \%$ patients after AEDs were withdrawn). The policy for postoperative AED reduction seems to affect the rates of both successful AED cessation and seizure recurrence.

Our patients took a mean of 2.1 types of AEDs before surgery and 1.2 types of AEDs after surgery. Temporal lobe resection effectively reduced the number of AEDs by one, over a mean follow-up period of 6.8 years. In a previous study, a mean of 1.7 drugs (677 AEDs/376 patients) used preoperatively was reduced to $1.0(243 / 244) 5$ years after surgery, and then further to $0.8(117 / 140) 10$ years after surgery. ${ }^{12)}$ This represented a mean reduction of 0.7 to 0.9 in the number of AEDs administered, which was about the same as the reduction observed in our patients. The mean number of preoperative AEDs in our series was 2.1 , which slightly exceeded the average in the previous series. This may be explained by the somewhat limited use of AEDs in Japan before 2007. In contrast to the more than 10 AEDs reported in the previous series, we used only 8 types of AEDs. The only new AEDs authorized for use in Japan during the study period were zonisamide, clobazam, and gabapentin.

Another purpose of this study was to identify the predictive factors associated with postoperative AED reduction, in order to provide better preoperative counseling for patients. We analyzed the factors associated with seizure-free outcome with successful AED cessation in patients who underwent temporal lobe resection. Short epilepsy duration turned out to be the only predictive factor for successful AED cessation. Previous studies analyzed factors related to AED cessation after patients obtained seizure-free outcome. Shorter disease duration seemed to affect successful AED cessation in seizure-free patients after temporal lobectomy.4) Several studies also identified younger age at surgery as a significant predictive factor for seizurefree status without drug treatment. ${ }^{1,4)}$ We found no 
significant relationship between age at surgery and postoperative drug cessation in our series, although the mean age at surgery was 26.8 years for patients without postoperative AEDs and 31.1 years for patients with postoperative AEDs. As we previously mentioned, the difference seems to stem mainly from the many inevitable biases among the different studies. However, most studies agree that early surgery may be a favorable predictor for seizure-free status after AEDs are discontinued. Neuronal kindling and secondary epileptogenesis may explain the higher rate of AED continuation in patients with longer disease duration or older age at surgery, and the superior outcome attained by early surgery. ${ }^{14)}$ The present study suggests that early surgical intervention after seizure onset is an important precondition for achieving seizure-free status without requiring AED treatment.

\section{References}

1) Al-Kaylani M, Konrad P, Lazenby B, Blumenkopf B, Abou-Khalil B: Seizure freedom off antiepileptic drugs after temporal lobe epilepsy surgery. Seizure 16: 95-98, 2007

2) Gilliam F, Kuzniecky R, Meador K, Martin R, Sawrie S, Viikinsalo M, Morawetz R, Faught E: Patientoriented outcome assessment after temporal lobectomy for refractory epilepsy. Neurology 53: 687-694, 1999

3) Griffin CT, Abastillas ME, Armon C, Lacanlale J, Liwnicz BH, Kaptain G, Dayes LA: Early antiepileptic drug reduction following anterior temporal lobectomy for medically intractable complex partial epilepsy. Seizure 13: 434-437, 2004

4) Kim YD, Heo K, Park SC, Huh K, Chang JW, Choi JU, Chung SS, Lee BI: Antiepileptic drug withdrawal after successful surgery for intractable temporal lobe epilepsy. Epilepsia 46: 251-257, 2005

5) Lee SY, Lee JY, Kim DW, Lee SK, Chung CK: Factors related to successful antiepileptic drug withdrawal after anterior temporal lobectomy for medial tem- poral lobe epilepsy. Seizure 17: 11-18, 2008

6) Lowe AJ, David E, Kilpatrick CJ, Matkovic Z, Cook MJ, Kaye A, O’Brien TJ: Epilepsy surgery for pathologically proven hippocampal sclerosis provides long-term seizure control and improved quality of life. Epilepsia 45: 237-242, 2004

7) McIntosh AM, Kalnins RM, Mitchell LA, Fabinyi GC, Briellmann RS, Berkovic SF: Temporal lobectomy: long-term seizure outcome, late recurrence and risks for seizure recurrence. Brain 127: 20182030, 2004

8) McIntosh AM, Wilson SJ, Berkovic SF: Seizure outcome after temporal lobectomy: current research practice and findings. Epilepsia 42: 1288-1307, 2001

9) Schmidt D, Loscher W: How effective is surgery to cure seizures in drug-resistant temporal lobe epilepsy? Epilepsy Res 56: 85-91, 2003

10) Shimizu H, Ohta Y, Suzuki I, Ishijima B, Sugishita M: Lateral temporal polar approach to mesial temporal lesions. Neurol Med Chir (Tokyo) 33: 360-364, 1993

11) Spencer DD: Anteromedial temporal lobectomy: directing the surgical approach to the pathologic substrate, in Spencer SS, Spencer DD (eds): Surgery for Epilepsy. Boston, Blackwell Scientific Publications, 1991, pp 129-148

12) Wieser HG, Hane A: Antiepileptic drug treatment before and after selective amygdalohippocampectomy. Epilepsy Res 55: 211-223, 2003

13) Wilson SJ, Saling MM, Kincade P, Bladin PF: Patient expectations of temporal lobe surgery. Epilepsia 39: 167-174, 1998

14) Yoon HH, Kwon HL, Mattson RH, Spencer DD, Spencer SS: Long-term seizure outcome in patients initially seizure-free after resective epilepsy surgery. Neurology 61: 445-450, 2003

Address reprint requests to: Taketoshi Maehara, MD, Department of Neurosurgery, Tokyo Medical and Dental University, 1-5-45 Yushima, Bunkyo-ku, Tokyo 113-8519, Japan.

e-mail: maehara.nsrg@tmd.ac.jp 